\title{
MAXIMAL TWO-SIDED IDEALS IN TENSOR PRODUCTS OF BANACH ALGEBRAS
}

\author{
KJELD B. LAURSEN
}

In [1] and [2] a construction of a bijection $\mathfrak{T M}_{3} \leftrightarrow \mathfrak{M}_{1} \times \mathfrak{T C}_{2}$ is given, where $\mathfrak{M}_{i}$ is the set of maximal modular (two-sided) ideals in the Banach algebra $A_{i}(i=1,2,3)$ and where $A_{3}=A_{1} \otimes_{\gamma} A_{2}$ is the greatest cross-norm tensor product of $A_{1}$ and $A_{2}$. In a recent correction [3] it is shown that there is indeed a closed 1-1 mapping $\mathfrak{N}_{1} \times \mathfrak{N}_{2} \rightarrow \mathfrak{T}_{3}$ when hull-kernel topologies are used. However, it is an open question when this mapping is surjective. In this note we show that the mapping is onto when one of the Banach algebras $A_{1}$ and $A_{2}$ is commutative. Also we give a correct proof of a theorem in [5], the original proof depended on [2].

The methods employed are adaptions of those in [6].

Suppose $A_{1}$ and $A_{2}$ are Banach algebras and suppose $A_{1}$ is commutative. Let $\mathfrak{M T}_{i}$ be the set of maximal modular (two-sided) ideals. Each $h \in \mathscr{T}_{1}$ is a continuous $C$-valued homomorphism and induces a homomorphism

$$
\phi_{h}: A_{3} \rightarrow A_{2}
$$

defined by

$$
t=\sum_{i=1}^{\infty} a_{1 i} \otimes a_{2 i} \in A_{3} \Rightarrow \phi_{h}(t)=\sum_{i=1}^{\infty} h\left(a_{1 i}\right) a_{2 i} \in A_{2} .
$$

Theorem 1. If $A_{1}, A_{2}, A_{3}=A_{1} \otimes A_{2}$ are Banach algebras with spaces $\mathfrak{T}_{i}$ of maximal modular ideals and if $A_{1}$ is commutative then there is a bijection

$$
\tilde{T}: \mathfrak{M}_{1} \times \mathfrak{M C}_{2} \rightarrow \mathfrak{M C}_{3} .
$$

This bijection is given by the following:

$M_{3} \subseteq A_{3}$ is an element of $\mathfrak{T}_{3}$ if and only if there is a continuous homomorphism $h \in \mathfrak{T C}_{1}$ and a maximal modular ideal $M_{2} \in \mathfrak{T}_{2}$ such that

$$
M_{3}=\phi_{h}^{-1}\left(M_{2}\right) \equiv \tilde{T}\left(h, M_{2}\right) .
$$

When the algebras have identities the mapping $\tilde{T}$ is identical with the one defined in [3] and, consequently, is closed with respect to the hull-kernel topologies.

Received by the editors January 29, 1969. 
Proof. Sufficiency. Suppose $h \in \mathscr{T}_{1}, M_{2} \in \mathscr{M}_{2}$ with identities modulo the ideal $u_{1}$ and $u_{2}$, respectively, and suppose $M_{3}=\phi_{h}^{-1}\left(M_{2}\right)$. Clearly, $M_{3}$ is a modular ideal with $u_{1} \otimes u_{2}$ as an identity modulo $M_{3}$. Suppose $M_{3}^{\prime} \supsetneqq M_{3}$. From the definition of $M_{3}$ we get $\phi_{h}\left(M_{3}^{\prime}\right) \supsetneqq M_{2}$, i.e. $\phi_{h}\left(M_{3}^{\prime}\right)=A_{2}$, since $M_{2}$ is maximal. Therefore, there is $t \in M_{3}^{\prime}$ so that $\phi_{h}(t)=u_{2}$. Now, $\phi_{h}\left(t-u_{1} \otimes u_{2}\right)=\phi_{h}(t)-h\left(u_{1}\right) u_{2}=u_{2}-u_{2}=0$ so $t-u_{1} \otimes u_{2}$ $\in M_{3} \subseteq M_{3}^{\prime}$; hence $u_{1} \otimes u_{2} \in M_{3}^{\prime}$ and $M_{3}^{\prime}=A_{3}$. It follows that $M_{3}$ is maximal.

To prove that every $M_{3} \in \mathbb{T}_{3}$ is of the form $\phi_{h}^{-1}\left(M_{2}\right)$ for some $h \in \mathscr{N}_{1}, M_{2} \in \mathfrak{N C}_{2}$ suppose first that $A_{1}$ and $A_{2}$ have identities, both denoted by $e$. Let $M_{3} \in \mathfrak{M}_{3}$. Let $\pi: A_{3} \rightarrow A_{3} / M_{3}$ be the canonical mapping and let $\pi_{i}$ be the restriction of $\pi$ to $A_{i}$ via the identifications

$$
A_{1} \leftrightarrow A_{1} \otimes e \subseteq A_{3}, \quad A_{2} \leftrightarrow e \otimes A_{2} \subseteq A_{3} .
$$

The kernel of $\pi_{1}$ is easily seen to be $A_{1} \cap M_{3}$ so in the diagram below provided us by the induced mapping theorem $U: A_{1} / A_{1} \cap M_{3} \rightarrow A_{3} / M_{3}$ is seen to be an isomorphism.

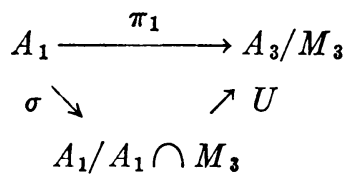

Since $A_{1}$ is commutative $\pi_{1}\left(A_{1}\right)$ is an abelian subalgebra of $A_{3} / M_{3}$. Since $\pi_{1}\left(A_{1}\right)$ and $\pi_{2}\left(A_{2}\right)$ commute elementwise, $\pi_{1}\left(A_{1}\right) \subseteq$ center $\left(\pi_{1}\left(A_{1}\right) \pi_{2}\left(A_{2}\right)\right)$. Consequently, from $A_{1} \otimes A_{2}$ being dense in $A_{1} \otimes{ }_{\gamma} A_{2}$ we infer that $\pi_{1}\left(A_{1}\right) \subseteq$ center $A_{3} / M_{3}$. From the diagram we then conclude $U\left(A_{1} / A_{1} \cap M_{3}\right)=U\left(\sigma\left(A_{1}\right)\right)=\pi_{1}\left(A_{1}\right) \subseteq \operatorname{center}\left(A_{3} / M_{3}\right)$. Therefore $A_{1} / A_{1} \cap M_{3} \cong C$ because $A_{3} / M_{3}$ is primitive [7, p. 61]. It follows that $A_{1} \cap M_{3}$ is a maximal ideal of $A_{1}$ and consequently is an element, $h$, of $\mathfrak{T r}_{1}$.

Next we show that kernel $\left(\phi_{h}\right) \subseteq M_{3}$. But this follows immediately from the observation that

$$
\pi=\pi_{2} \circ \phi_{h}
$$

together with kernel $(\pi)=M_{3}$. $\left(\pi=\pi_{2} \circ \phi_{h}\right.$ is seen to hold by noting that both sides of the equation are continuous and coincide on $A_{1} \otimes A_{2}$, i.e. everywhere.)

We now show that $M_{3}=\phi_{h}^{-1}\left(M_{2}\right)$ where $M_{2} \in \Re_{2}$. Since $\phi_{h}$ is surjective, $\phi_{h}\left(M_{3}\right)$ is an ideal in $A_{2}$. If $\phi_{h}\left(M_{3}\right)=A_{2}$ then there is $t \in M_{3}$ so that $\phi_{h}(t)=e \in A_{2}$ and hence $\phi_{h}(t-e \otimes e)=0$. Since kernel $\left(\phi_{h}\right) \subseteq M_{8}$, $e \otimes e \in M_{3}$, i.e. $M_{3}=A_{3}$. It follows that $\phi_{h}\left(M_{3}\right)$ is a proper ideal. Let 
$M_{2} \supseteq \phi_{h}\left(M_{3}\right)$ be a maximal ideal. We claim that $M_{3}=\phi_{h}^{-1}\left(M_{2}\right)$. Since $M_{3}$ as well as $\phi_{h}^{-1}\left(M_{2}\right)$ are maximal (see the sufficiency part of this proof) it suffices to note that $M_{3} \subseteq \phi_{h}^{-1}\left(M_{2}\right)$.

Before removing the assumption concerning the presence of identities we show that the mapping defined above is identical with that of [3].

Let $\digamma_{i}$ be the set of closed (two-sided) ideals in $A_{i}(i=1,2,3)$. Then Gelbaum defines the following mappings $S$ and $T$.

If $I_{i} \in \mathscr{F}_{i}(i=1,2)$ then

$$
\begin{aligned}
R: A_{3} \ni t & =\sum a_{1 i} \otimes a_{2 i} \rightarrow \sum\left(a_{1 i} / I_{1}\right) \otimes\left(a_{2 i} / I_{2}\right) \\
& \in A_{1} / I_{1} \otimes_{\gamma} A_{2} / I_{2}
\end{aligned}
$$

and $T\left(I_{1}, I_{2}\right)=$ kernel $(R) \in \mathcal{F}_{3}$.

If $I_{3} \in \mathcal{F}_{3}$ then

$$
\begin{gathered}
G_{1}: A_{1} \ni a_{1} \rightarrow a_{1} \otimes e / I_{3} \in A_{3} / I_{3}, \\
G_{2}: A_{2} \ni a_{2} \rightarrow e \otimes a_{2} / I_{3} \in A_{3} / I_{3} ;
\end{gathered}
$$

kernel $\left(G_{i}\right)=I_{i}, \quad i=1,2$ and $S\left(I_{3}\right)=\left(I_{1}, I_{2}\right) \in F_{1} \times F_{2}$. Note that $S\left(I_{3}\right)=\left(I_{3} \cap A_{1} \otimes e, I_{3} \cap e \otimes A_{2}\right)$.

It is then shown that $S T$ is the identity on $\mathfrak{M}_{1} \times \mathfrak{N}_{2}$ and that $S$ induces a mapping $\tilde{S}: \mathfrak{M}_{3} \rightarrow \mathscr{N}_{1} \times \mathfrak{M}_{2}$, while $T$ induces $\tilde{T}: \mathfrak{M}_{1} \times \mathfrak{M}_{2} \rightarrow \mathfrak{M}_{3}$ with the property that $\tilde{S} \widetilde{T}$ is the identity on $\mathfrak{M}_{1} \times \Re_{2}$. Actually $\tilde{T}$ and $\tilde{S}$ are defined as choice functions; if $\left(M_{1}, M_{2}\right) \in \mathfrak{N}_{1} \times \mathbb{N}_{2}$ then $T\left(M_{1}, M_{2}\right) \in \mathcal{F}_{3}$. Take any $M_{3} \in \Re_{3}$ such that $M_{3} \supseteq T\left(M_{1}, M_{2}\right)$ and define $\tilde{T}\left(M_{1}, M_{2}\right)=M_{3}$. Similarly, if $M_{3} \in \mathscr{T}_{3}$ and $S\left(M_{3}\right)=\left(I_{1}, I_{2}\right) \in \mathcal{F}_{1}$ $\times F_{2}$ pick $M_{i} \in \mathscr{T M}_{i}$ such that $I_{i} \subseteq M_{i}(i=1,2)$ and define $\widetilde{S}\left(M_{3}\right)$ $=\left(M_{1}, M_{2}\right)$. We shall show that in our situation $T\left(h, M_{2}\right)=\phi_{h}^{-1}\left(M_{2}\right)$ for every $\left(h, M_{2}\right) \in \mathscr{T l}_{1} \times \Re_{2}$; this will show that $\tilde{T}$ is surjective and that $\tilde{S}=\widetilde{T}^{-1}$. Let $\left(h, M_{2}\right) \in \mathfrak{M}_{1} \times \mathfrak{T}_{2}$; then $T\left(h, M_{2}\right)=\operatorname{kernel}(R)$, where

$$
\begin{aligned}
R: t & =\sum a_{1 i} \otimes a_{2 i} \rightarrow \sum a_{1 i} / h \otimes a_{2 i} / M_{2} \\
& =\sum h\left(a_{1 i}\right) a_{2 i} / M_{2} \\
& =\left(\sum h\left(a_{1 i}\right) a_{2 i}\right) / M_{2} \\
& =\phi_{h}(t) / M_{2},
\end{aligned}
$$

i.e. $t \in T\left(h, M_{2}\right)$ if and only if $\phi_{h}(t) \in M_{2}$ so

$$
T\left(h, M_{2}\right)=\phi_{h}^{-1}\left(M_{2}\right) \text {. }
$$

It follows that $M_{3}=T\left(h, M_{2}\right) \in \mathscr{T}_{3}$. We have shown that Gelbaum's 
mapping $\tilde{T}$ is identical with that introduced here. Theorem 1 [3] gives us the result that $\widetilde{T}$ is closed with respect to the hull-kernel topologies.

If $A_{1}$ and $A_{2}$ do not necessarily have identities, let $\tilde{A}_{i}(i=1,2)$ be the smallest algebra with an identity containing $A_{i}$ and let $\widetilde{A}_{3}=\tilde{A}_{1}$ $\otimes_{\gamma} \tilde{A}_{2}$. Then $A_{3}$ can be viewed as an ideal in $\tilde{A}_{3}[2$, Lemma 2]. What remains to be shown is that if $M_{3} \in \mathscr{N}_{3}$ then we can find $h \in \mathscr{M}_{1}$ and $M_{2} \in \mathscr{M}_{2}$ such that $M_{3}=\phi_{h}^{-1}\left(M_{2}\right)$. Let $M_{3} \in \mathscr{N}_{3}$ and let $u$ be an identity $\bmod M_{3}$. Define

$$
\tilde{M}_{3}=\left\{t \in \tilde{A}_{3}, u t \in M_{3} \text { and } t u \in M_{3}\right\} .
$$

Then the following holds:

(a) $M_{3} \subseteq \tilde{M}_{3}$, and

(b) $u$ is an identity (in $\tilde{A}_{3}$ ) $\bmod \tilde{M}_{3}$. (a) is clear, and (b) is proved in [2, Lemma 4].

Let $\tilde{M}_{3}^{\prime}$ be a maximal modular ideal containing $\tilde{M}_{3}$. Since $\tilde{M}_{3}^{\prime}$ is proper $u \notin \tilde{M}_{3}^{\prime}$. By the part already proved we can find maximal modular ideals $\tilde{h} \subseteq \tilde{A}_{1}$ and $\tilde{M}_{2} \subseteq \tilde{A}_{2}$ such that

$$
\tilde{M}_{3}^{\prime}=\phi \tilde{\tilde{h}}^{-1}\left(M_{2}\right) \text {. }
$$

If $\tilde{h} \supseteq A_{1}$ or if $\tilde{M}_{2} \supseteq A_{2}$ then $\phi_{\tilde{h}}(u)=0$, i.e. $u \in \tilde{M}_{3}^{\prime}$ which is a contradiction. It follows that $\tilde{h} \cap A_{1} \equiv h \in \mathfrak{M}_{1}$ and $\tilde{M}_{2} \cap A_{2} \equiv M_{2} \in \mathfrak{M}_{2}$. Left to show is that

$$
M_{3}=\phi_{h}^{-1}\left(M_{2}\right) .
$$

Both sides of this expression are maximal ideals so it suffices to show

but

$$
M_{3} \subseteq \phi_{h}^{-1}\left(M_{2}\right) ;
$$

$$
\begin{aligned}
t \in M_{3} & \Rightarrow t \in A_{3} \cap \tilde{M}_{3}^{\prime} \\
& \Rightarrow \phi_{h}(t) \in \tilde{M}_{2} \cap A_{2}=M_{2} .
\end{aligned}
$$

Also $\phi_{\tilde{h}}(t)=\phi_{h}(t)$, since $h=\tilde{h} \cap A_{1}$, so

$$
\phi_{h}\left(M_{3}\right) \subseteq M_{2} \text { i.e. } M_{3} \subseteq \bar{\phi}_{h}^{-1}\left(M_{2}\right) .
$$

This finishes the proof.

A Banach algebra is strongly semisimple if the intersection of all maximal modular two-sided ideals is $\{0\}$.

Let $\lambda$ be the "least cross-norm" [8] and let $\tau: A_{1} \otimes_{\gamma} A_{2} \rightarrow A_{1} \otimes_{\lambda} A_{2}$ be the canonical mapping (the extension to all of $A_{1} \otimes_{\gamma} A_{2}$ of the identity on the algebraic tensor product $A_{1} \otimes A_{2}$ ). 
Theorem 2 [5]. Suppose $A_{1}$ and $A_{2}$ are strongly semisimple Banach algebras and suppose $A_{1}$ is commutative. Then $A_{1} \otimes_{\gamma} A_{2}$ is strongly semisimple if and only if

$$
\tau: A_{1} \otimes_{\gamma} A_{2} \rightarrow A_{1} \otimes_{\lambda} A_{2}
$$

is $1-1$.

Proof. Suppose $\tau$ is $1-1$. Since $A_{1}$ is commutative, Theorem 1 tells us that Theorem 2 [3] applies so $A_{3}=A_{1} \otimes_{\gamma} A_{2}$ is strongly semisimple. Suppose conversely that $A_{1} \otimes_{\gamma} A_{2}$ is strongly semisimple. Then if $t \in A_{3}$ and $t \neq 0$ we can find $M_{3} \in \mathbb{N}_{3}$ so that $t \notin M_{3}=\phi_{h}^{-1}\left(M_{2}\right)$ for some $\left(h, M_{2}\right) \in \mathscr{M}_{1} \times \mathfrak{M}_{2}$ (by Theorem 1). Consequently, $\phi_{h}(t) \notin M_{2}$ and in particular $\phi_{h}(t) \neq 0$ so there is $a_{2}^{*} \in A_{2}^{*}$ (the dual space of $A_{2}$ ) for which $a_{2}^{*}\left(\phi_{h}(t)\right) \neq 0$. Since $\lambda(\tau(t))=\sup \left|a_{1}^{*} \otimes a_{2}^{*}(t)\right|$ where the supremum is taken when $a_{i}^{*}$ ranges over the unit ball of $A_{i}^{*}(i=1,2)$ and since $a_{2}^{*}\left(\phi_{h}(t)\right)=h \otimes a_{2}^{*}(t)$, as an easy computation shows, we conclude that $\lambda(\tau(t)) \neq 0$ and hence that $\tau$ is $1-1$.

Remark. It is easy to see that if $A_{1}$ is commutative and $A_{1} \otimes_{\gamma} A_{2}$ is strongly semisimple then $A_{1}$ and $A_{2}$ are strongly semisimple: $a_{2} \in \bigcap_{M_{2} \in \mathfrak{T}_{2}} M_{2}$ if and only if $\phi_{h}^{-1}\left(a_{2}\right)=0$ for every $h \in \mathscr{T}_{1}$ by Theorem 1 and the strong semisimplicity of $A_{1} \otimes_{\gamma} A_{2}$. Since $\phi_{h}\left(a_{1} \otimes a_{2}\right)=h_{1}\left(a_{1}\right) a_{2}$ it follows that $a_{1} \otimes a_{2}=0$ for any $a_{1} \in A_{1}$, i.e. $a_{2}=0$. Similarly, $a_{1} \in \bigcap_{h \in \Re_{1}} h$ implies that $\phi_{h}\left(a_{1} \otimes a_{2}\right)=0$ for every $h \in \mathfrak{N}_{1}$ and every $a_{2} \in A_{2}$, i.e. $a_{1} \otimes a_{2}=0$ by the strong semisimplicity of $A_{1} \otimes_{\gamma} A_{2}$ so $a_{1}=0$.

Corollary 1. Let $G$ be a locally compact abelian group, $A$ a Banach algebra and $L^{1}(G, A)$ the algebra of Bochner-integrable functions from $G$ to $A$ with convolution multiplication. Then $L^{1}(G, A)$ is strongly semisimple if and only if $A$ is strongly semisimple.

Proof. $L^{1}(G, A)=L^{1}(G) \otimes_{\gamma} A[4]$. Moreover, $\tau: L^{1}(G) \otimes_{\gamma} A \rightarrow L^{1}(G)$ $\otimes_{\lambda} A$ is 1-1 since $L^{1}(G)$ satisfies Grothendieck's condition of approximation [4]. The sufficiency then follows from Theorem 2, since $L^{1}(G)$ is known to be (strongly) semisimple [9]. The necessity is covered by the above remark.

Corollary 2 [9, Theorem 1.7]. Let $G$ be an abelian locally compact group and $H$ a compact group. Then $L^{1}(G \times H)$ is strongly semisimple.

Proof. $L^{1}(G \times H)=L^{1}(G) \otimes_{\gamma} L^{1}(H)[4]$ and $L^{1}(H)$ is strongly semisimple [9]. 


\section{REFERENCES}

1. B. R. Gelbaum, Tensor products of Banach algebras, Canad. J. Math. 11 (1959), 297-310. MR 21 \#2922.

2. - Note on the tensor product of Banach algebras, Proc. Amer. Math. Soc. 12(1961), 750-757. MR 24 \#A1039.

3. - Tensor products of Banach algebras, Corrections of earlier work, 1968 (preprint).

4. A. Grothendieck, Produits tensoriels topologiques et espaces nucleaires, Mem. Amer. Math. Soc. No. 16 (1955), MR 17, 763.

5. K. B. Laursen, Tensor products of Banach-*-algebras, Thesis, University of Minnesota, Minneapolis, Minn., 1967.

6. A. Lebow, Maximal ideals in tensor products of Banach algebras, Bull. Amer. Math. Soc. 74 (1968), 1020-1022. MR 38 \#539.

7. C. Rickart, General theory of Banach algebras, The University Series in Higher Mathematics, Van Nostrand, Princeton, N. J., 1960. MR 22 \#5903.

8. R. Schatten, $A$ theory of cross-spaces, Ann. of Math. Studies, no. 26, Princeton Univ. Press, Princeton, N. J., 1950. MR 12, 186.

9. I. E. Segal, The group algebra of a locally compact group, Trans. Amer. Math. Soc. 61 (1947), 69-105. MR 8, 438.

University of Aarhus, Aarhus, Denmark 